\title{
Analysis of selected allergic reactions among psoriatic patients
}

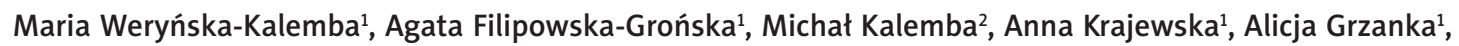 \\ Andrzej Bożek ${ }^{1}$, Jerzy Jarząb ${ }^{1}$
}

${ }^{1}$ Chair and Clinical Department of Internal Diseases, Dermatology and Allergology in Zabrze, Medical University of Silesia, Katowice, Poland

${ }^{2}$ Nuclear Medicine and Endocrine Oncology Department, Maria Sklodowska-Curie Memorial Cancer Center and Institute of Oncology, Gliwice Branch, Gliwice, Poland

Adv Dermatol Allergol 2016; XXXIII (1): 18-22

DOI: 10.5114/pdia.2014.44015

\begin{abstract}
Introduction: Psoriasis is a chronic and recurrent inflammatory skin disease. The aetiology is still unknown in spite of numerous scientific researches. There is very little evidence which does not provide enough knowledge about allergic reactions in psoriatic patients. Based on the fact that the epidermal barrier damage allows different allergen types to penetrate into deep layers of epidermis and skin, we can assume that it may lead to immunological reactions.

Aim: To investigate the allergic reaction indicators and hypersensitivity assessment about contact, inhalant and food allergens. The results were analysed with regard to clinical disease indicators and progression stage of dermal lesions.

Material and methods: Eighty patients with psoriasis were examined. The concentration of total IgE antibodies and allergen specific IgE antibodies (aslgE) were analysed. Standard epidermal tests and atopy patch tests were performed. All the patients were estimated for their dermatological condition based on the PASI scale. The control group consisted of 50 patients without psoriasis and allergic history.

Results: Significantly higher concentration of total E immunoglobulin has been stated in the patients with psoriasis. Higher concentrations of specific allergic IgE antibodies were more often observed in the examined group but the most frequently observed values were present in 1-3 class. The most common airborne allergens were birch, artemisia, timothy and rye pollens. There have not been any significant statistical differences in the case of positive epidermal test results.

Conclusions: There is slightly expressed hypersensitivity in psoriatic patients. This hypersensitivity degree correlates with the intensification of symptoms.
\end{abstract}

Key words: psoriasis, immunoglobulin E, allergic reactions.

\section{Introduction}

Psoriasis is a chronic and recurrent inflammatory skin disease. It is assumed that it affects $2-3 \%$ of the whole population [1]. The latest research shows the psoriasis complexity as a chronic disease characterized by a systemic inflammatory process concerning not only the skin. In spite of some promising research focused on pathogenesis, new directions and therapeutic possibilities, psoriasis still remains a chronic disease with vague aetiology. Genetic, immunological and environmental factors play an important role in the psoriasis etiopathogen- esis [2-5]. A lot of endo- and exogenous factors may play a role in the appearance of pathologic changes, especially in patients with a genetic predisposition. Disorders connected with immunological reactions are the basis of the psoriasis process. This process is initiated by the keratinocyte proliferation with the help of stimulated lymphocytes. Continuous interaction between cells of the immunological system and cytokines creates a self-running mechanism which is responsible for the creation and support of the pathological changes. Disease complexity makes a reflection about allergic diseases coexistence with psoriasis. A long-term disease process may lead to

Address for correspondence: Maria Weryńska-Kalemba MD, PhD, Chair and Clinical Department of Internal Diseases, Dermatology and Allergology in Zabrze, Medical University of Silesia, M. Skłodowskiej 10, 41-800 Zabrze, Poland, phone: +48 32 2713165, +48 664968021 , e-mail: maria.werynska@gmail.com

Received: 14.01.2014, accepted: 21.03.2014. 
the epidermal barrier damage which leads to a higher allergen exposure [6]. Antigens penetration by pathologically changed skin may also be treated as an additional element of the disease progression. Literature data on serum IgE, contact hypersensitivity in patients suffering from psoriasis are limited and often controversial.

\section{Aim}

The aim of this work is to estimate hypersensitivity concerning contact, inhalant and food allergens in patients with psoriasis. The analysis of the research results has been carried out with reference to the clinical picture of the disease - location, morphology, and progression stage of the dermal lesion.

\section{Material and methods}

The examined group consisted of 80 patients with psoriasis of the Clinic Department of Internal Diseases, Dermatology and Allergology of the Medical University of Silesia in Zabrze. The examination was conducted in accordance with the Helsinki Declaration after the acceptance of the local Bioethical Association (Bioethical Association of the Medical University of Silesia, Katowice KNW/0022/KB1/82/1/09). All the patients were 18-60 years old. Moreover, all of them were estimated for their dermatological condition based on the PASI scale (Psoriasis Area and Severity Index). The concentration of total IgE antibodies and allergen-specific antibodies (aslgE) was evaluated, standard epidermal tests and atopic patch tests within the skin without pathological changes were conducted. Atopic patch tests (Stallergenes) contained allergenic extracts of dust mites, mouldy fungus, birch pollen, motherwort, grass and cat's and dog's hairs, were put on the healthy back skin and checked after 48 and 72 h. The European Standard Allergen Set was also performed in similar conditions. All the patients had quantitative determination of the total IgE concentration in serum by ELISA - the immunoenzymatic method (Allergopharma). The reference value of total IgE immunoglobulin was below $100 \mathrm{kU} / \mathrm{l}$. The concentration of IgE against inhalant and food allergens were marked by fluoroimmunoenzymatic method (Euroimmun). The tested allergens included timothy, rye, birch, motherwort, cat, dog, horse, dust mites, moulds, egg, milk, cod, wheat flour, rice, soya, hazelnut, carrot, and potato. The estimation of the IgE antibodies class concentration was based on a class division in a 6 -grade scale. The concentration in 2-6 class was defined as clinically crucial (0.7-100 kU/l).

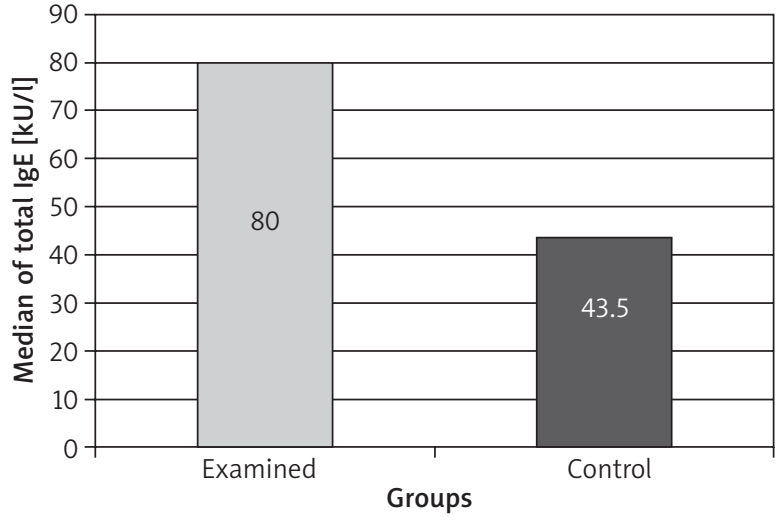

Figure 1. Median of IgE concentration

The control group consisted of 50 persons without psoriasis and allergy history.

\section{Statistical analysis}

The statistical analysis was conducted using 6.0 Statistica program. The results were presented using descriptive statistical parameters such as median, average value and standard deviation. Compatibility of variable and standard distribution was checked by Shapiro-Wilk test. In order to compare the results, we used U MannWhitney and Kruskal-Wallis tests. To determine the number of differences in groups, $\chi^{2}$ test was used. Moreover, to specify the relation between tested indicators, we used the order correlation ranks test according to Spearman. The significance level was $p<0.05$.

\section{Results}

\section{Total immunoglobulin E concentration}

The median of the total value of IgE class antibodies was higher in the tested group in comparison to the control group: 80 ( $\min 3.2$; $\max 672$ ) kU/l and 43.5 (min 3.7; $\max 225) \mathrm{kU} / \mathrm{l}$, respectively. The difference was statistically significant ( $p<0.05$, U Mann-Whitney test) and is presented in Figure 1 and Table 1.

\section{Correlation of total IgE concentration with psoriasis intensification (PASI)}

The median of total IgE concentration in patients with PASI 1-5 was 49.5 ( $\min 6.9$; $\max 89$ ) kU/l, with PASI 5-10 was 60.9 ( $\min 3.24 ; \max 273) \mathrm{kU} / \mathrm{l}$, but with PASI 10-20 it was 131.5 ( $\min 23.36$; $\max 672$ ) kU/l. Total IgE concentration correlated with PASI $(r=0.648 ; p<0.05)$ - Figures 2 and 3.

Table 1. Total IgE concentration ( $\mathrm{kU} / \mathrm{l})$ in the tested and control group

\begin{tabular}{lccccc}
\hline Group & Patients number & Median & Range & Average SD & Value of $p$ \\
\hline Tested & 80 & 80 & $3.24-672$ & $107.69 \pm 101.5$ & $<0.05$ \\
\hline Control & 50 & 43.5 & $7-140$ & $56 \pm 47.5$ & $<0.05$ \\
\hline
\end{tabular}




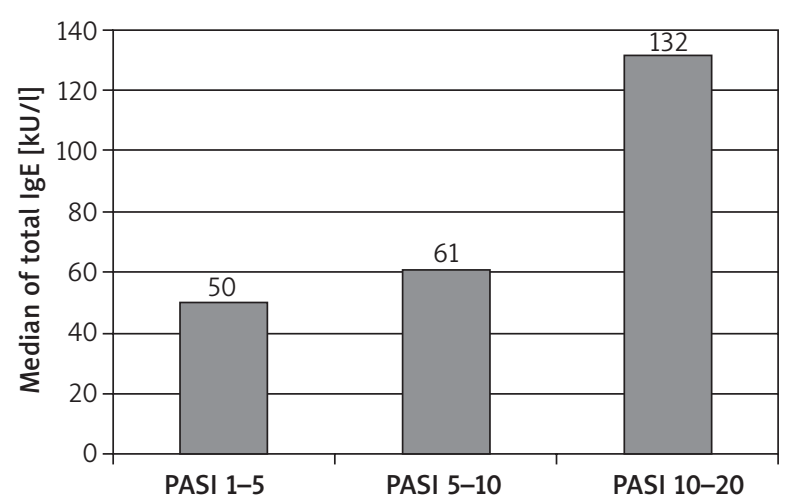

Figure 2. Total IgE in the case of psoriasis symptoms intensity

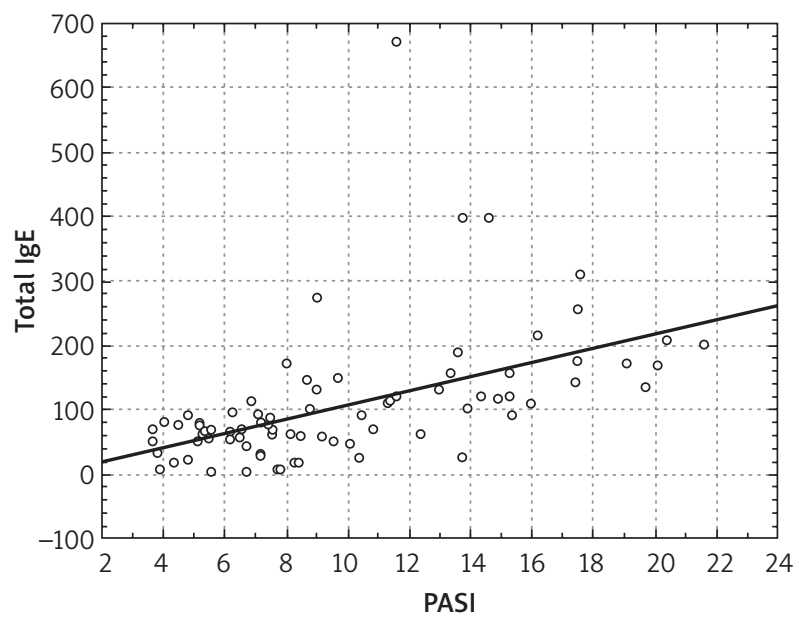

Figure 3. Correlation between total IgE concentration and PASI

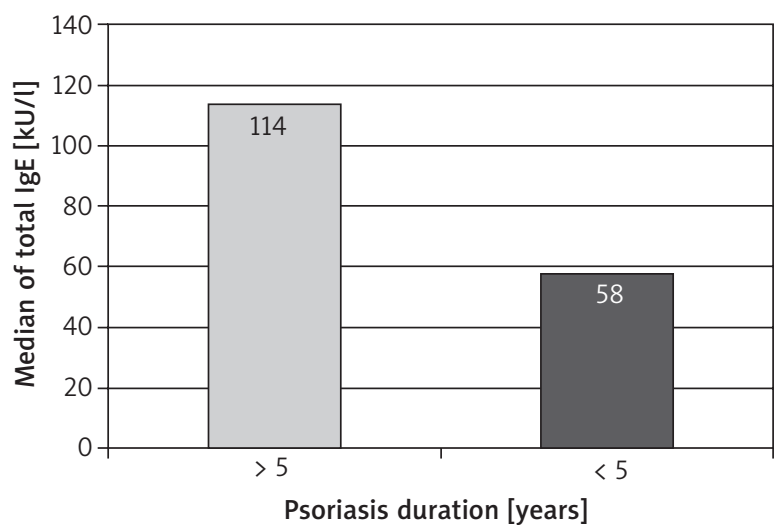

Figure 4. Total IgE concentration in patients with shorter and longer psoriasis duration

Total E immunoglobulin concentration and disease duration

A higher total lgE concentration was observed in the group of patients with a longer period of skin changes (above 5 years). Its median was $114 \mathrm{kU} / \mathrm{l}$ while in the group with a shorter disease duration it was $57.7 \mathrm{kU} / \mathrm{l}$. The total IgE concentration and psoriasis duration is presented in Figure 4.

\section{AslgE concentration in the examined group and control group: airborne allergens, food allergens}

A higher concentration of allergy-specific lgE antibodies was more often observed in the examined group in comparison to the control group but the most frequently observed values were present in 1-3 class. The most common airborne allergens were birch, timothy and rye pollens. A higher concentration of IgE related to Dermatophagoides pteronyssinus, Cladosporium herbarium and Alternaria alternata was observed (Figures 5-7).

\section{Hypersensitivity to the most common contact allergens}

There were no statistically significant differences between frequency of positive results of epidermal tests with reference to the control group. This situation concerns the European Standard and atopic patch tests.

\section{Discussion}

Within the last years, some issues concerning the existence of various antibody classes in the case of patients with psoriasis including lgE antibodies, responsible for atopic reactions have been already discussed [7-11]. In previous studies, serum levels of total IgE were significantly higher in psoriasis patients than in controls (42\% vs. $10 \%$; $p<0.05$ ) [12]. It was observed in psoriasis that a total $\mathrm{E}$ immunoglobulin concentration was higher in patients with a more serious disease clinical picture, e.g. psoriatic erythroderma. Li's research showed the serum IgE level was found to be elevated in $81.3 \%$ of patients with psoriatic erythroderma in comparison to $6.3 \%$ of psoriasis vulgaris patients as controls [13]. Our study shows that this phenomenon may occur in patients who suffer from mild and moderate disease and the antibody titre correlates with intensity of dermal changes (PASI scale). In Ding's research, the levels of IgE were analysed in generalized pustular psoriasis and psoriasis vulgaris patients. The percentage of patients with pustular psoriasis who had increased IgE levels was significantly higher than that of patients with psoriasis vulgaris [14]. The total IgE concentration and allergy-specific IgE concentration in our study was related to the intensification of disease symptoms. The most common airborne allergens were pollens of birch, motherwort, rye and timothy. The presence of a higher IgE concentration against food allergens such as potato and carrot was observed. It is worth emphasising that in this case we can observe cross reactions e.g. birch and motherwort pollens can cross with potato or carrot allergens. The research concerning contact hypersensitivity in psoriasis patients 


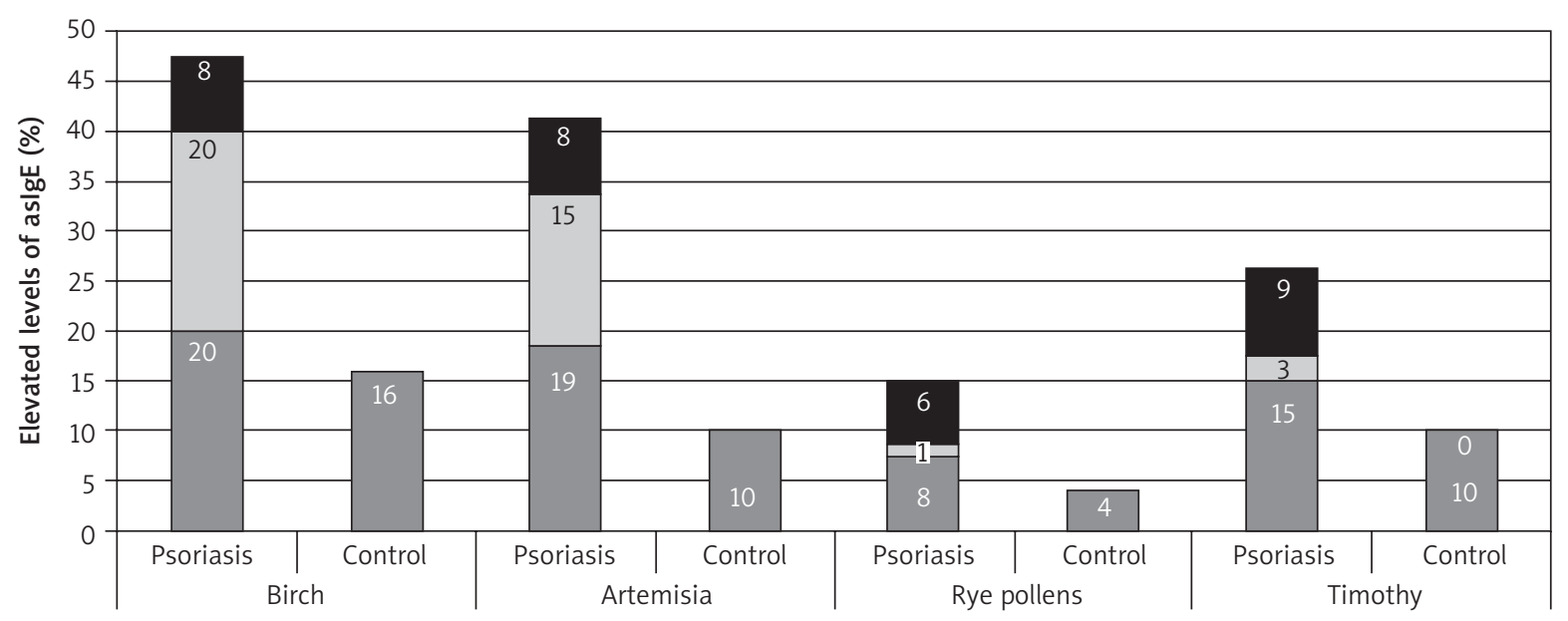

Air-derived allergens

- Level $\geq 3$ aLevel 2 口Level 1

Figure 5. Allergy-specific E immunoglobulin concentration against airborne allergens

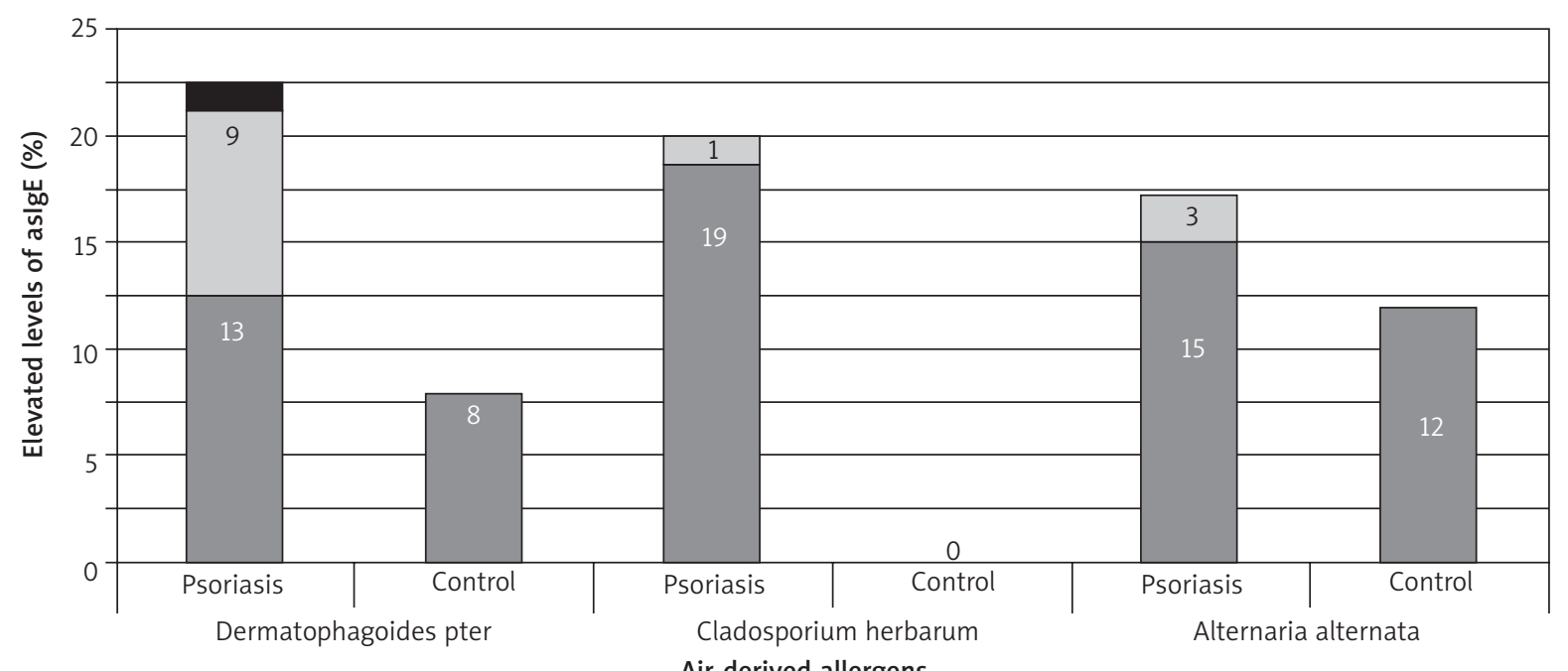
Air-derived allergens

- Level $\geq 3 \quad$ aLevel $2 \quad \square$ Level 1

Figure 6. Percentage of higher allergy-specific E immunoglobulin concentration - dust and moulds

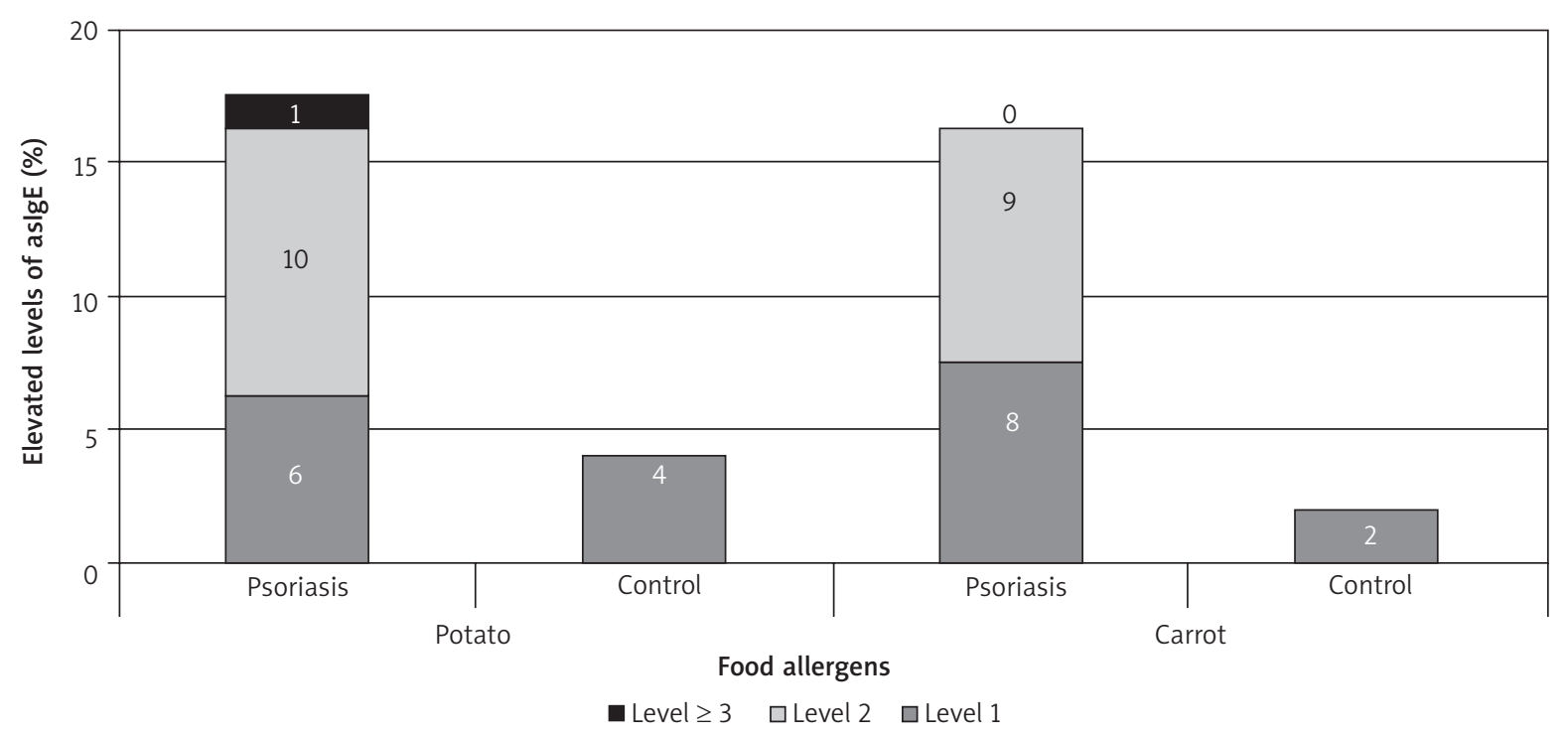

Figure 7. AslgE-food allergens 
have not shown univocal results. There are some weakly expressed data about contact hypersensitivity in psoriasis patients contrary to strongly expressed hypersensitivity connected with contact allergens in patients with palmoplantar pustulosis [15-17]. Our research shows that there is weakly expressed contact hypersensitivity in psoriasis patients. More common were: nickel sulphate, cobalt chloride and lanolin. In Fleming's research, we can find very often: nickel, aromatic mixture, birch tar, rosin and neomycin. However, any significant differences concerning the results were noted $[18,19]$. Bangsgaard's research showed weaker contact hypersensitivity in psoriasis with reference to the control group without psoriasis [20]. Compatible research results show the complexity of this phenomenon. Some authors suggest that incorrect T lymphocytes function may influence the handicapped reaction to the allergens [21]. Moreover, a disordered function of Langerhans cells in the epidermis may have some influence on the skin reaction to contact allergens [22]. There is also some evidence about the reverse relation between some autoimmunological diseases and contact allergy [23-25]. Allergy diagnosis requires accuracy and appropriate selection of psoriasis patients. We expected some technical or interpretation problems in those patients. However, we did not observe any atypical skin reactions or Koebner phenomena. Taking into consideration a big amount of allergic reactions in the general population (20-40\%), we should remember that the same number may concern psoriasis patients. Data from the ECAP programme show that we belong to one of the most "allergic" societies worldwide. Even $40 \%$ of people declare this fact which is dependent on the region and gender [26]. Allergy, similarly to psoriasis, is a serious problem for educational and professional efficiency.

\section{Conclusions}

Weakly expressed hypersensitivity to some inhalant, food and contact allergens in patients suffering from psoriasis is not strong enough to conclude about the connection between allergy reactions and psoriasis pathogenesis. However, the hypersensitivity correlates with intensification of the disease symptoms.

\section{Conflict of interest}

The authors declare no conflict of interest.

\section{References}

1. Buchau AS, Gallo RL. Innate immunity and antimicrobial defense systems in psoriasis. Clin Dermatol 2007; 25: 616-24.

2. Crissey JT, Parish LC. Two hundred years of dermatology. J Am Acad Dermatol 1998; 39: 1002-6.

3. Nedoszytko B, Sokołowska-Wojdyło M, RuckemannDziurdzińska K, et al. Chemokines and cytokines network in the pathogenesis of the inflammatory skin diseases: atopic dermatitis, psoriasis and skin mastocytosis. Adv Dermatol Allergol 2014; 31: 84-91.
4. Maciejewska-Radomska A, Szczerkowska-Dobosz A, Rębała K, et al. Frequency of streptococcal upper respiratory tract infections and $\mathrm{HLA}-\mathrm{CW}^{\star} 06$ allele in 70 patients with guttate psoriasis from northern Poland. Adv Dermatol Allergol 2015; 32: 455-8.

5. Stawczyk-Macieja M, Szczerkowska-Dobosz A, Rębała K, Purzycka-Bohdan D. Genetic background of skin barrier dysfunction in the pathogenesis of psoriasis vulgaris. Adv Dermatol Allergol 2015; 32: 123-6.

6. Segre JA. Epidermal barrier formation and recovery in skin disorders. J Clin Invest 2006; 116: 1150-8.

7. Holgate ST, Church MK, Lichtenstein LM. Alergia. Czelej, Lublin 2003.

8. Kulick KB, Mogavero H, Provost TT, et al. Serologic studies in patients with lupus erythematosus and psoriasis. J Am Acad Dermatol 1983; 8: 631-4.

9. Negosanti M, Fanti PA, Gasponi A, et al. IgE serum levels in psoriasis. Dermatologica 1981; 163: 474-5.

10. Przybilla B, Ring J, Volk M. Total IgE levels in the serum in dermatologic diseases. Hautarzt 1986; 37: 77-82.

11. Jarząb J. Interpretacja oznaczeń immunoglobuliny $E$ w chorobach alergicznych. In: Postępy w alergologii. Vol. II. Płusa T (ed.). Medpress, Warsaw 1997.

12. Ovcina-Kurtovic N, Kasumagic-Halilovic E. Serum levels of total immunoglobulin $\mathrm{E}$ in patients with psoriasis: relationship with clinical type of disease. Med Arh 2010; 64: 28-9.

13. Li LF, Sujan S, Yang H, et al. Serum immunoglobulins in psoriatic erythroderma. Clin Exp Dermatol 2005; 30: 125-7.

14. Ding Y, Yi X, Yu N. Serum IgE levels are increased in patients with generalized pustular psoriasis. Clin Exp Dermatol 2013; 38: 549-52.

15. Heule F, Tahapary GJM, Belloc R, et al. Delayed-type hypersensitivity to contact allergens in psoriasis: a clinical evaluation. Contact Dermatitis 1998; 38: 78-82.

16. Caca-Biljanovska N, Vlckova-Laskoska M, Balbanova-Stefanova $M$, et al. Frequency of delayed-type hypersensitivity to contact allergens in palmo-plantar psoriasis. Prilozi 2005; 26: 131-41.

17. Yiannias JA, Winkelmann RK, Connoly SM. Contact sensitivities in palmar plantar pustulosis (acropustulosis). Contact Dermatitis 1998; 39: 108-11.

18. Fleming CJ, Burden AD. Contact allergy in psoriasis. Contact Dermatitis 1997; 36: 274-6.

19. Barile M, Cozzani E, Anonide A, et al. Is contact allergy rare in psoriatics? Contact Dermatitis 1996; 35: 113-4.

20. Bangsgaard N, Engkilde K, Thyssen JP, et al. Inverse relationship between contact allergy and psoriasis: results from a patient- and population-based study. Contact Dermatitis 1996; 35: 113-4.

21. Moss C, Friedman PS, Shuster S. Impaired contact hypersensitivity in untreated psoriasis and the effects of the fotochemotherapy and dihydranol/UVB. Br J Dermatol 1981; 105: 503-8.

22. Cumberbatch $M$, Singh $M$, Deaman RJ, et al. Impaired Langerhans cell migration in psoriasis. J Exp Med 2006; 203: 953-60.

23. Engkilde K, Menne T, Johansen JD. Inverse association between rheumatoid arthritis and allergic contact dermatitis. Contact Dermatitis 2008; 58 (Suppl. 1): 68-9.

24. Engkilde K, Menne T, Johansen JD. Inflamatory bowel disease in relation to contact allergy: a patient-base study. Scand J Gastroenterol 2007; 42: 572-6.

25. Engkilde K, Menne T, Johansen JD. Inverse relationship between allergic contact dermatitis and type I diabetes mellitus: a retrospective clinic-based study. Diabetologica 2006; 49: 644-7.

26. Samoliński B, Raciborski F, Tomaszewska A, et al. Epidemiology of allergic diseases in Poland - ECAP study. Allergy 2008; 88: 626-7. 